\title{
RMC (Renal Medullary Cancer): A Case Report and
}

\section{Literature Review}

\author{
Patrícia Taranto ${ }^{1}$, Rodrigo Coutinho Mariano ${ }^{1}$, Daniel Eiger ${ }^{1}$, Gustavo Schvartsman ${ }^{2}$, Samy Tarnovschi ${ }^{1}$, Renee \\ Zon Filippi $^{1}$ and Andrey Soares ${ }^{1}$ \\ 1. Hospital Israelita Albert Einstein (HIAE), São Paulo (SP) 05651901, Brazil \\ 2. University of Texas MD Anderson Cancer Center (MDACC), Houston 77030, Texas, USA
}

\begin{abstract}
RMC (renal medullary carcinoma) is considered a rare entity, corresponding to approximately $2 \%$ of primary kidney tumors, usually occurring in the second decade of the patient's life and it is closely related with previous history of sickle cell disease. Studies have reported RMC as highly aggressive, poorly responsive to chemotherapy, surgery, radiotherapy or targeted therapy. This disease's median overall survival is less than 12 months. We are reporting a case of a 21 -year-old woman with sickle cell trait, whose initial clinical presentation was RMC already metastatic to the liver. She underwent total right nephrectomy, and her clinical picture worsened during hospitalization with pneumonia, bowel obstruction, oliguria and death in less than two months after diagnosis, with no performance status to attempt any treatment options to her cancer at any time. Although some cases in the literature had reported good response to neoadjuvant therapy, this disease has early recurrence and little response to treatment. Our objective is to call the attention for this aggressive desease to estimulate new perspectives and further studies with chemotherapy and other different kinds of treatment, mainly because only surgical intervention is an incomplete aproach.
\end{abstract}

Key words: Kidney neoplasms, RMC (renal medullary carcinoma), carcinoma, renal cell, sickle cell trait.

\section{Introduction}

RMC (renal medullary cancer) was first described in 1995. It is considered a rare entity, corresponding to approximately $2 \%$ of primary kidney tumors that occurs primarily in young male, in the second decade of life, particularly in patients affected by haemoglobinopathies (especially sickle cell anemia [1, 2]). It harbors distinct histological features, despite sometimes being considered a subtype of collecting duct carcinoma [3]. Case reports suggest its extreme aggressiveness, usually presenting as metastatic disease at diagnosis. It is poorly responsive to chemotherapy, radiotherapy, targeted therapy and to surgery, with a median overall survival of less than 12 months [4]. Rathmell and Monk [5] described a case series in which the use of ddMVAC (dose dense methotrexate, vinblastine, doxorubicin and cisplatin)

Corresponding author: Patrícia Taranto, research fellow, research field: oncology. in three patients produced a significant benefit in symptom palliation and survival [5]. Another series performed karyotyping evaluation of four patients, showing all had chromosome 11 monosomy and two of them had chromosome 3 abnormalities [6].

This is a case report of a 21-year-old female patient with sickle cell trait and family members with sickle cell disease, who presented with de novo metastatic RMC to the liver. She was submitted to a total right nephrectomy but unfortunately developed clinical deterioration due to a pneumonia and bowel obstruction, ultimately leading to oliguria and death in less than two months, without active treatment to the cancer.

\section{Case Report}

This is a 21-year-old female patient with sickle cell trait and family members with sickle cell anemia, who presented with weight loss (28 pounds in six months), right flank pain and an abdominal mass on physical 


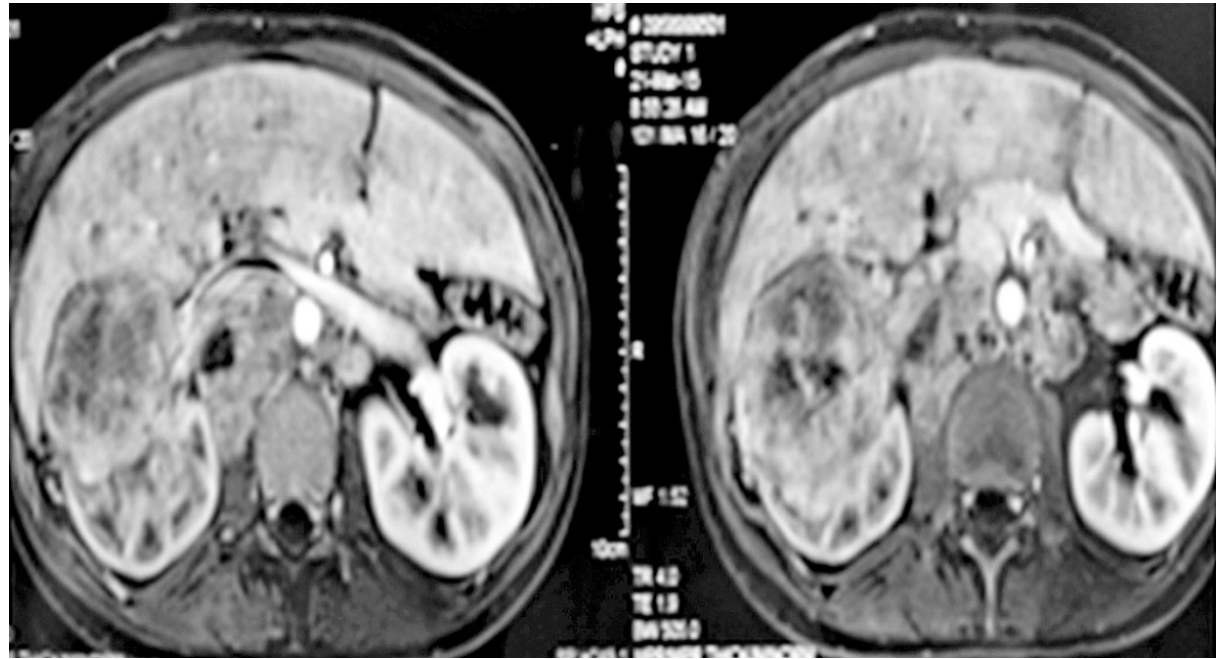

Fig. 1 Abdominal MRI showing an expansive right kidney lesion.

exam. MRI (magnetic resonance imaging) of the abdomen showed a $7.6 \mathrm{~cm}$ mass in the right kidney, associated with nodules of up to $1.4 \mathrm{~cm}$ in the perirenal space suggestive of implants, and extensive heterogeneous nodal enlargement of up to $4 \mathrm{~cm}$ in the right retrocural chain and adjacent to the inferior vena cava (Fig. 1). Furthermore, the liver was enlarged with sparse focal lesions of up to $2 \mathrm{~cm}$.

The patient was submitted to a total right nephrectomy, but in the post-operative setting developed a nosocomial pneumonia with pleural effusion, requiring broad-spectrum antibiotics and multiple pleural drainages in the ICU (intensive care unit). Right pleurodesis was performed and pleural effusion cytology was negative for neoplastic cells. She developed abdominal distension followed by nausea and vomiting, compatible with intestinal subocclusion, with no improvement with clinical management. Due to hemodynamic instability, surgery was not feasible. She rapidly progressed with worsening of renal function and died less than two months after diagnosis.

\section{Pathological Findings}

The surgical specimen consisted of a neoplasia measuring $7 \mathrm{~cm}$ in its largest axis, with ill-defined borders, infiltration of the renal pelvis, fat in the renal sinus and its surrounding fat tissue (Fig. 2).
Noncontiguous invasion to the ipsilateral adrenal gland and to its surrounding fat tissue was found. Surgical margins were compromised.

On microscopic evaluation, characteristic epithelial cells with eosinophilic cytoplasm, large nuclei and prominent nucleolus were seen with solid, tubular and reticular growing features. An intense desmoplastic reaction was showed in the stroma and a mixed inflammatory infiltrate surrounding the neoplastic cells was evident (Fig. 3).

By immunohistochemical staining, the neoplastic cells were positive for PAX-8, OCT-4, EMA and pan-cytokeratin, and had loss of the immunoreactivity for INI-1 (Fig. 4).

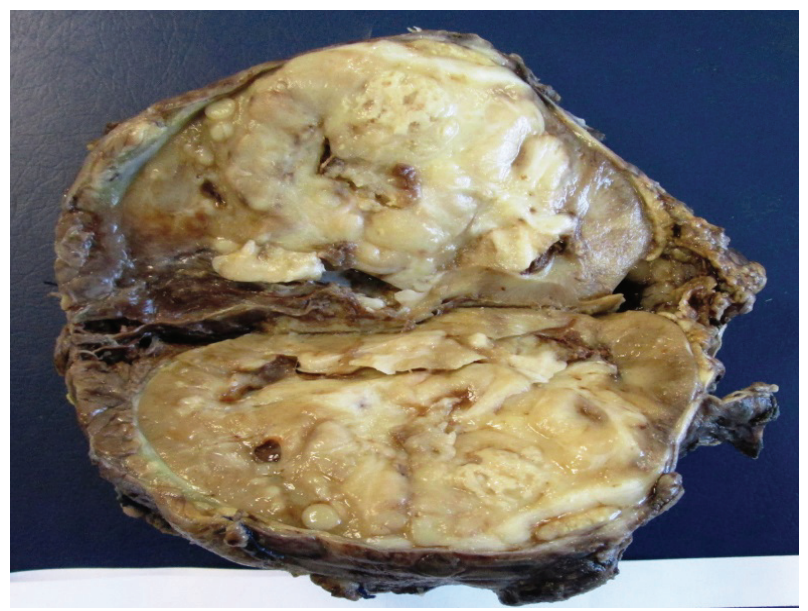

Fig. 2 A picture of the macroscopic exam of the surgical specimen. 


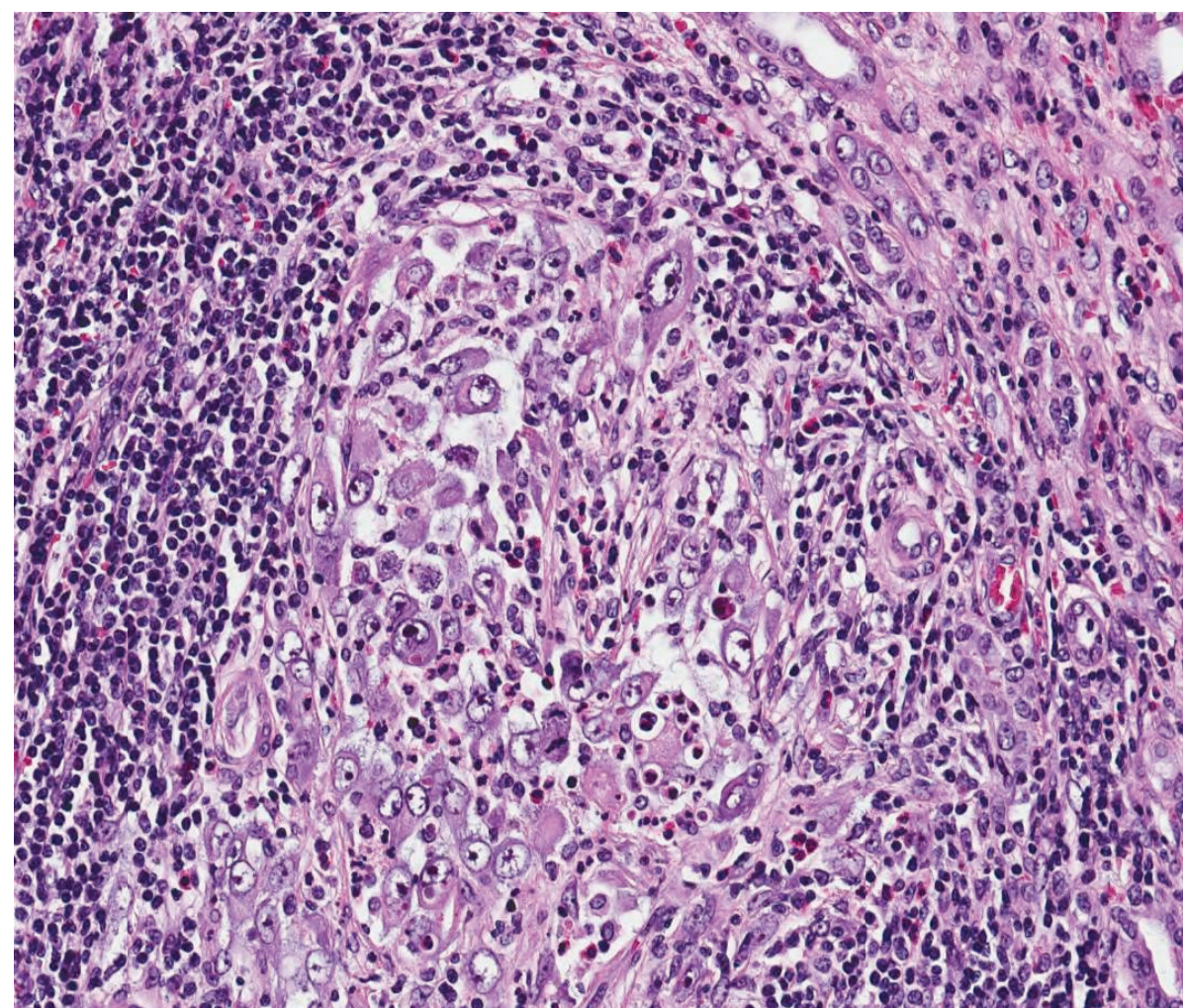

Fig. 3 A microscopic picture of the tumor on H.E. staining, showing it was constituted of cells with large nuclei and prominent nucleolus surrounded by a mixed inflammatory infiltrate.

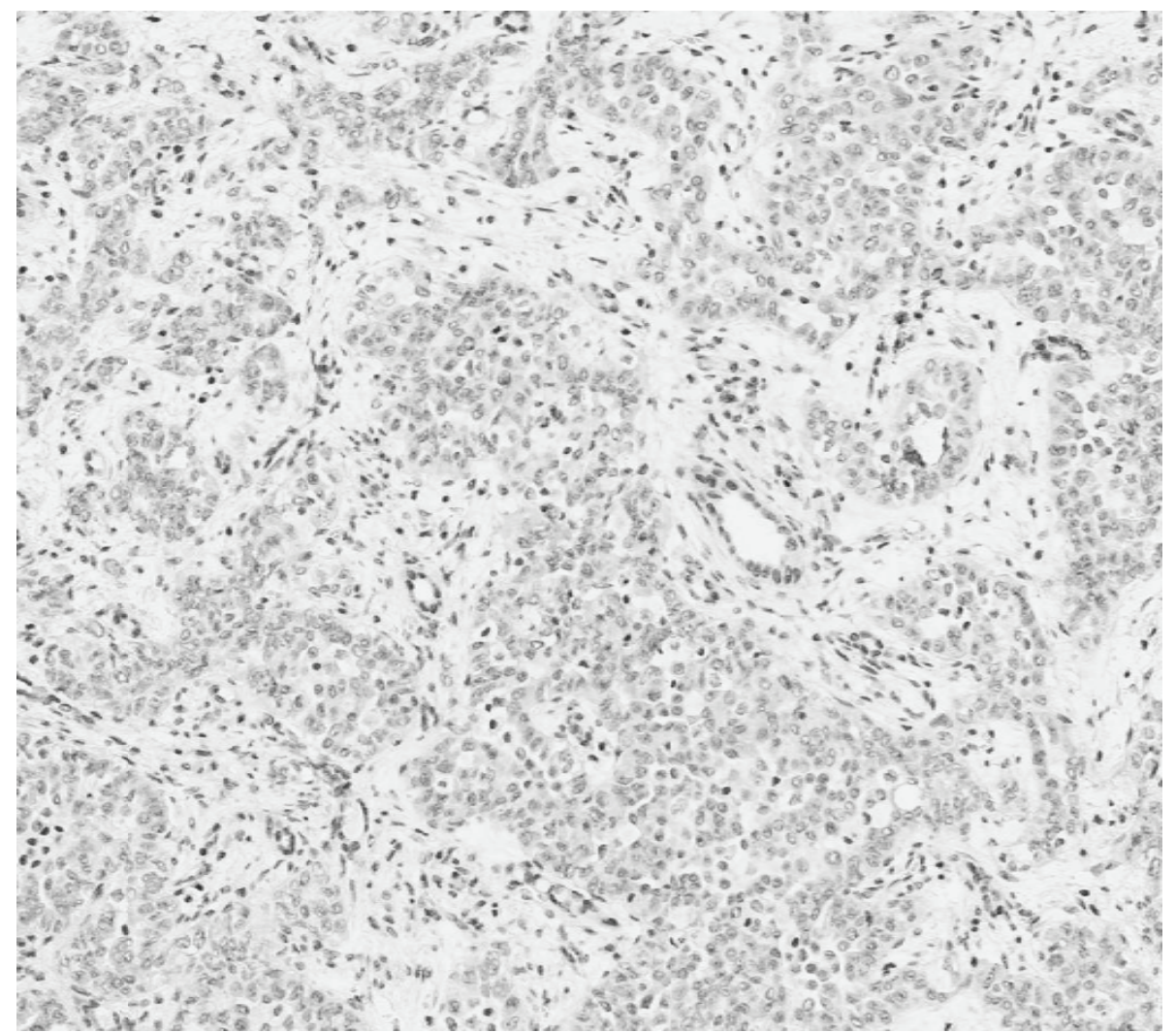

Fig. 4 A microscopic picture of the immunoreactivity for INI-1, showing that it was absent on the neoplastic cells. 


\section{Discussion and Conclusions}

Initially described by Berman [7] in 1975 as the seventh nephropathy associated with sickle cell anemia, RMC was interpreted as a picture of major hematuria, papillae necrosis, nephrotic syndrome, renal infarction, hipoestenuria and pyelonephritis [7]. Davis et al. [8] reported in 1995 a series of 34 cases of an aggressive neoplasia with peculiar microscopic features probably originating in the collecting ducts of the kidneys in young patients with sickle cell anemia, therefore describing this new disease, "medullary carcinoma of the kidney", as the seventh sickle cell anemia nephropathy [8].

This neoplasm occurs mostly in young black males, especially in those with sickle cell trait (instead of the anemia per se) [9]. It is usually incidentally detected, with clinical features resembling those of classic kidney carcinoma, such as flank pain, palpable mass, frank hematuria, weight loss and a predilection for the right side. The majority of patients present with de novo metastatic disease, especially to regional lymph nodes, adrenal glands, lungs, liver, inferior vena cava e peritoneum [10], and have a very aggressive disease course. Commonly, it shows no response to standard therapies, leading to a poor prognosis and median overall survival of less than 12 months [2]. Another series of cases from the Bronx institution reports nine patients with RMC, being all of them with sickle cell trait, male to female ratio of 2:1 and age at diagnosis between 13 to 31 years. They all presented with flank pain, two thirds presented with hematuria and one third with a palpable mass. Eight of nine evaluated patients had masses that varied in size between $4 \mathrm{~cm}$ to $12 \mathrm{~cm}$. Our patient had similar characteristics, being 21 years old at diagnosis, with the aforementioned clinical symptoms and a $7.6 \mathrm{~cm}$ right kidney mass.

Despite these tumors not having any specific radiological features, on the right clinical ground they can be suspected on scans as an invasive mass that is deep in the renal parenchyma, renal pelvis and sinusoids, with kidney enlargement but without alteration of its shape [11].

While the tumor pathogenicity is not completely understood, it is known that there is an association with sickle cell nephropathy and its cytogenetic abnormalities, such as triploidy with chromosome 11 monosomy, translocation $(3 ; 8) \quad(\mathrm{p} 21 ; \mathrm{q} 24)$ and tetraploid with the bcr/abl translocation in the chromosomes 9 and 22 [12-20]. Some evidences suggest that the short arm of chromosome 11 (11p), where the beta-globin gene is located, might be involved in the tumor pathogenicity, too [13].

Macroscopically it is characterized as a tumor that grows in the renal medulla with extension to the pelvis and chalices [8]. Small satellite nodules can be found in the normal cortex and near soft tissues. Histologically, it is characterized by a tubulopapillary architecture with a dark eosinophilic cytoplasm, desmoplasia, high grade nuclear cytology, prominent nucleolus [3], multiple hemorrhagic areas with extensive necrosis. In the majority of cases, lymphovascular invasion can be observed, with a massive inflammatory infiltrate and abundant neutrophils [1]. By immunohistochemistry analysis, Swartzel at al. [3] reported 15 RMC specimens, where all had positivity for the epithelial marker CAM5.5 and for epithelial membrane antigens, with absent staining for cytokeratin 34EE12. Cytokeratins 7 and 20 and carcinoembryonic antigen had variable expression [3]. Despite the negative result in our patient specimen, a new histochemical marker called INI-1 was recently described for RMC. Loss of SMARCB1/INI-1, a tumor suppressor gene, was initially described on rhabdoid pediatric tumors of the central nervous system, kidney and soft tissues. More recently, a complete loss of immunoexpression of SMARCB/INI-1 was found in $15 \%$ of collecting duct tumors, with no differences in clinicopathological features or in endpoints related to the positivity of the marker, which suits it for the differential diagnosis of RMC [14]. Keratins AE1/AE3 and EMA are usually 
positive, whereas for CEA the degree of positivity is lower [15].

The available therapeutic modalities are poorly studied, including surgery, chemotherapy, radiotherapy and biological drugs. The majority of patients have a low response rate and regimens are based on the treatment of urothelial carcinomas [16]. Some authors reports treatment based on platinum salts [17], anthracyclines [18] and Bortezomib [19]. Simpson et al. [20] reviewed RMC cases published between 1995 and 2003 and described 28 patients with a median overall survival of 32 weeks (range: 2 to 68 weeks), and the patients who lived the longest were exposed to MVAC regimen.

According to the Brazilian experience, the best responses found where with platinum-based treatments. Molecular-targeting agents might be promising but patients treated with sunitinib had no responses [4].

Recently, Amjad et al. [21] reported a case where a 23-year-old African American patient with sickle cell anemia had a near complete clinical and pathological response to ddMVAC. This particular patient had six cycles of neoadjuvant ddMVAC with great tolerance and no need of hospitalization, and then proceeded with a total nephrectomy with retroperitoneal lymphadenectomy, showing a near complete pathological response, developing recurrence only 16 months later [21]. Another case report from Alabama University described an 11-year-old African American patient who was submitted to three cycles of neoadjuvant carboplatin, gemcitabine and paclitaxel, followed by a right nephrectomy with an aortocaval lymphadenectomy and multiple nodule resections of the lungs, with no evidence of diseases on the surgical specimens of the lymph nodes and pulmonary nodules. Six additional cycles of the same chemotherapy treatment were delivered, with good tolerance and a disease-free survival of 11 months. Eventually, the patient developed headaches and focal neurological signs, which turned out to be leptomeningeal involvement, followed by death 24 months after the initial diagnosis [22].

In the era of guided therapy and knowledge about driver mutation, a case report showed a patient treated with Everolimus (an MTOR inhibitor) maintenance after induction chemotherapy regimen of PCG (Paclitaxel Cisplatin-Gemcitabine) based on results of PTEN deficiency expressed in molecular profiling and next generation sequencing. This patient responded to induction therapy, had complete remission and remained in remission for seven month [23]. Other potential new drugs to be considered are histone deacetylase inhibitors, which have shown activity against malignant rhabdoid tumor cell lines [24]. and the ALK inhibitor crizotinib [25]..

Accordingly to the current epidemiology and disease description in the literature, RMC is an extremely rare and aggressive disease, presenting mostly in young patients with sickle cell trait or overt anemia. Our patient was diagnosed in her 20 s, with an expansive $7.6 \mathrm{~cm}$ mass in the right kidney and hepatic metastasis. She underwent a radical nephrectomy but unfortunately developed multiple post-op complications and clinical deterioration, eventually dying two months later without receiving any systemic treatment. When facing such an aggressive and rare clinical picture, strong suspicion and early diagnosis are of major concern to improve this already grim prognosis carried by these young sickle cell patients. Despite some reports of favorable responses to neoadjuvant therapy, the disease tends to recur early in its course, with a paucity of effective therapeutic options thereafter, and still remains an unmet need for new perspectives and investigation of chemotherapeutical interventions, since surgery itself is an incomplete and inadequate isolated treatment option.

\section{References}

[1] Baig, M. A., Lin, Y. S., Rasheed, J., and Mittman, N. 2006. "Renal Medullary Carcinoma." J. Natl. Med. Assoc. 98: 1171-4.

[2] Hakimi, A. A., Koi, P. T., Milhoua, P. M., Blitman, N. M., 
Li, M., Hugec, V., et al. 2007. "Renal Medullary Carcinoma: The Bronx Experience." Urology 70 (5): 878-82.

[3] Swartz, M. A., Karth, J., Schneider, D. T., Rodriguez, R., Beckwith, J. B., and Perlman, E. J. 2002. "Renal Medullary Carcinoma: Clinical, Pathologic, Immunohistochemical, and Genetic Analysis with Pathogenetic Implications." Urology 60 (6): 1083-9.

[4] Maroja Silvino, M. C., Venchiarutti Moniz, C. M., Munhoz Piotto, G. H., Siqueira, S., Galapo Kann, A., and Dzik, C. 2013. "Renal Medullary Carcinoma Response to Chemotherapy: A Referral Center Experience in Brazil." Rare Tumors 5 (3): e44.

[5] Rathmell, W. K., and Monk, J. P. 2008. "High-Dose-Intensity MVAC Foradvanced Renal Medullary Carcinoma: Report of Three Cases and Literature Review." Urology 72 (3): 659-63.

[6] Avery, R. A., Harris, J. E., Davis Jr., C. J., Borgaonkar, D. S., Byrd, J. C., and Weiss, R. B. 1996. "Renal Medullary Carcinoma: Clinical and Therapeutic Aspects of a Newly Described Tumor." Cancer 78 (1): 128-32.

[7] Berman, L. B. 1974. "Sickle Cell Nephropathy." JAMA 228 (10): 1279.

[8] Davis, C. J., Mostofi, F. K., and Sesterhenn, I. A. 1995. "Renal Medullary Carcinoma. The Seventh Sickle Cell Nephropathy." Am. J. Surg. Pathol. 19: 1-11.

[9] Wesche, W. A., Wilmas, J., Khare, V., and Parham, D. M. 1998. "Renal Medullary Carcinoma: A Potential Sickle Cell Nephropathy of Children and Adolescents." Pediatr. Pathol. Lab. Med. 18: 97-113.

[10] Patel, K., Livni, N., and Macdonald, D. 2006. "Renal Medullary Carcinoma, a Rare Cause of Hematuria in Sickle Cell Trait." Br. J. Hoematol. 132 (1): 1.

[11] Davidson, A. J., Choyke, P. L., Hartman, D. S., and Davis, C. J. 1995. "Renal Medullary Carcinoma Associated with Sickle Cell Trait: Radiographic Findings.” Radiology 195 (1): 83-5.

[12] Stahischmidt, J., Cullinane, C., Roberts, P., and Picton, S. 1999. "Renal Medullary Carcinoma: Prolonged Remission with Chemotherapy, Immunohistochemical Characterization and Evidence of bcr/abl Rearrangement." Med. Pediatr. Oncol. 33: 551-7.

[13] Avery, R. A., Harris, J. E., Davis, C. J., Borgaonkar, D. S., Byrd, J. C., and Weiss, R. B. 1996. "Renal Medullary Carcinoma: Clinical and Therapeutic Aspects of a Newly Described Tumor." Cancer 78: 128-32.

[14] Elwood, H., Chaux, A., Schultz, L., Illei, P. B., Baydar, D. E., Billis A., et al. 2011. "Immunohistochemical Analysis of SMARCB1/INI-1 Expression in Collecting Duct
Carcinoma." Urology 78 (2): 474.e1-5.

[15] Watanabe, I. C., Billis, A., Guimarães, M. S., Alvarenga, M., De Matos, A. C., Cardinalli, I. A., et al. 2007. "Renal Medullary Carcinoma: Report of Seven Cases from Brazil." Mod. Pathol. 20 (9): 914-20.

[16] Baig, M. A., Lin, Y. S., Rasheed, J., and Mittman, N. 2006. "Renal Medullary Carcinoma." J. Natl. Med. Assoc. 98: 1171-4.

[17] Strouse, J. J., Spevak, M., Mack, A. K., Arceci, R. J., Small, D., Loeb, D. M. 2005. "Significant Responses to Platinum-Based Chemotherapy in Renal Medullary Carcinoma." Pediatr. Blood Cancer 44: 407-11.

[18] Schaeffer, E. M., Guzzo, T. J., Furge, K. A., Netto, G., Westphal, M., Dykema, K., et al. 2010. "Renal Medullary Carcinoma: Molecular, Pathological and Clinical Evidence for Treatment with Topoisomerase-Inhibiting Therapy." BJU Int. 106:62-5.

[19] Ronnen, E. A., Kondagunta, G. V., and Motzer, R. J. 2006. "Medullary Renal Cell Carcinoma and Response to Therapy with Bortezomib.” J. Clin. Oncol. 24 (9): e14.

[20] Simpson, L., He, X., Pins, M., Huang, X., Campbell, S. C., Yang, X. J., et al. 2005. "Renal Medullary Carcinoma and ABL Gene Amplification.” J. Urol. 173 (6): 1883-8.

[21] Amjad, A. I., Ali, H., Appleman, L. J., Maranchie, J., Jackman, S., Parwani, A., et al. 2014. "Renal Medullary Carcinoma: Case Report of an Aggressive Malignancy with Near-Complete Response to Dose-Dense Methotrexate, Vinblastine, Doxorubicin, and Cisplatin Chemotherapy." Case Reports in Oncological Medicine 2014: 615895.

[22] Walsh, A., Kelly, D. R., Vaid, Y. N., Hilliard, L. M., and Friedman, G. K. 2010. "Complete Response to Carboplatin, Gemcitabine, and Paclitaxel in a Patient with Advanced Metastatic Renal Medullary Carcinoma." Pediatr. Blood Cancer 55: 1217-20.

[23] Lipkin, J. S., Rizvi, S. M., Gatalica, Z., Sarwani, N. E., Holder, S. L., Kaag, M., et al. 2015. "Therapeutic Approach Guided by Genetic Alteration: Use of MTOR Inhibitor in Renal Medullary Carcinoma with Loss of PTEN Expression." Cancer Biology \& Therapy 16 (1): 28-33.

[24] Furchert, S. E., Lanvers-Kaminsky, C., Juurgens, H., Jung, M., Loidl, A., and Fruhwald, M. C. 2007. "Inhibitors of Histone Deacetylases as Potential Therapeutics Tools for High-Risk Embryonal Tumors of the Nervous System of Childhood." Int. J. Cancer 120: 1787-94.

[25] Alvarez, O., Rodriguez, M. M., Jordan, L., and Sarnaik, S. 2015. "Renal Medullary Carcinoma and Sickle Cell Trait: A Systematic Review.” Pediatr. Blood Cancer 62: 1694-9. 